\title{
Original
}

\section{Expression of Signaling Molecules Related to Wnt Pathway in Cleft Palate Induced by Retinoic Acid during Perinatal Stage}

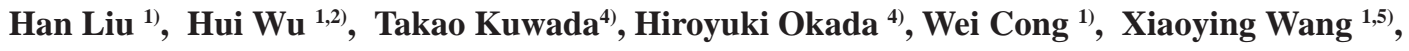 \\ Guangwei Sun ${ }^{3)}$, Xiaoxi Xu ${ }^{3)}$, Toshiro Sakae ${ }^{4)}$ and Jing Xiao ${ }^{1)}$ \\ ${ }^{1)}$ Department of Oral Biology, College of Stomatology, Dalian Medical University, Dalian, China \\ 2) Department of Stomatology, Hainan Medical Collage Hospital,Haikou, China \\ 3) Dalian Institute of Chemical Physics, Chinese Academy of Sciences,Dalian, China \\ ${ }^{4}$ Department of Histology,Nihon University School of Dentistry at Matsudo, Matsudo, Chiba, Japan \\ ${ }^{5}$ Department of Stomatology, China National Pwtroleum Corporation Central Hospital, Langfang, China \\ (Accepted for publication, February 21, 2012)
}

\begin{abstract}
Signaling pathways have been shown to participate in the process of Retinoic acid (RA) - induced cleft

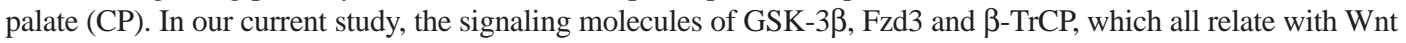
pathway, were screened from the palate tissues of the RA-induced CP in mice by the Gene-chip Technology. But, their expression pattern and level in palates during perinatal stages have not been known yet. In the studies, mRNA level of GSK-3 $\beta$, Fzd3 and $\beta$-TrCP were detected by quantitative real-time PCR, and showed significant difference between the Embryonic day 18 (ED18) before birth and Postnatal day 0 (PD0) after birth, as well as between the RA-

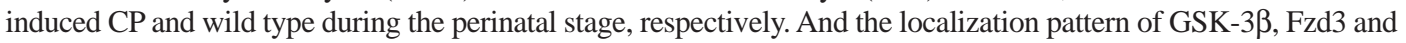
$\beta$-TrCP proteins in palates was also characterized. Our data indicates that the Wnt signaling pathway may involve in the RA-induced cleft palate during peirnatal stage.
\end{abstract}

Key Words: Retinoic acid, Cleft palate, Wnt Signals, Animal model, Perinatal stage

\section{Introduction}

Cleft palate (CP) is one of the most common congenital abnormalities in human being. It is considered to be a kind of multigenetic and susceptible disease, in which the deleterious genetic background is combined with environmental factors. The environmental factors may induce the gene mutation and control the proliferation, differentiation and migration of cells that contribute to the formation of cleft palate. All-trans retinoic acid (atRA), the derivative of vitamin $\mathrm{A}$, has been indentified as an important environmental factor in teratogenic effect ${ }^{1}$. The mechanism of RA-induced CP is to inhibit the proliferation of embryonic palate mesenchyme (EPM) cells through interfering DNA synthesis. The function of RA are achieved by binding to its receptors on the cellular membrane , and transfer the signals to the nucleus by combined with its binding proteins in the cytoplasm, and then regulates its down- stream gene transcription factors in the nucleus.

Wnt signaling pathway plays essential roles in the epithelial-

Correspondence to: Dr. Jing Xiao, Department of Oral Biology, College of Stomatology, Dalian Medical University, 9 Western Section, Lvshun South Street, Dalian, 116044, China. Phone: 0086-411-8611-0137 E-mail: xiaoj@dlmedu.edu.cn mesenchymal interaction and out growth of facial prominence during embryonic orofacial development. They are a large family of secreted glycoprotein with 19 members present in mammals, which encoded by Wnt gene ${ }^{2)}$. The $\beta$-Catenin-mediated Wnt canonical (Wnt/ $\beta$-Catenin) signaling pathway plays a pivotal role in activating target gene by stable the level of $\beta$-Catenin. Wnt/ $\beta$ Catenin signaling cascade is activated by WNT ligand, such as wnt1, wnt3a and wnt8. When Wnt proteins bind the cell-surface receptors, Frizzled and lipoprotein receptor-related (LRP) 5/6 to form the coreceptor, the coreceptor can activate the scaffold protein Dishevelled (Dvl) which encoded by Dsh gene family by combine with a conserved sequence ${ }^{3)}$. GSK-3 $\beta$ phosphorylation is suppressed through Hyperphosphorylating Dishevelled, and leading to the high dose level of $\beta$-Catenin in the cytoplasm. Stabilized $\beta$-Catenin enters the nucleus and interacts with the nuclear transcriptional factors, such as T-cell factor/lymphoid enhancer factor (TCF/LEF), to regulate the given gene transcription and decide the differentiation, polarity, proliferation, and migration of cells.

Glycogen synthase kinase-3 (GSK-3) is a multifunctional serine/threonine kinase. In mammal, there are two GSK-3 isoforms 
J.Hard Tissue Biology Vol. 21(2):173-180, 2012

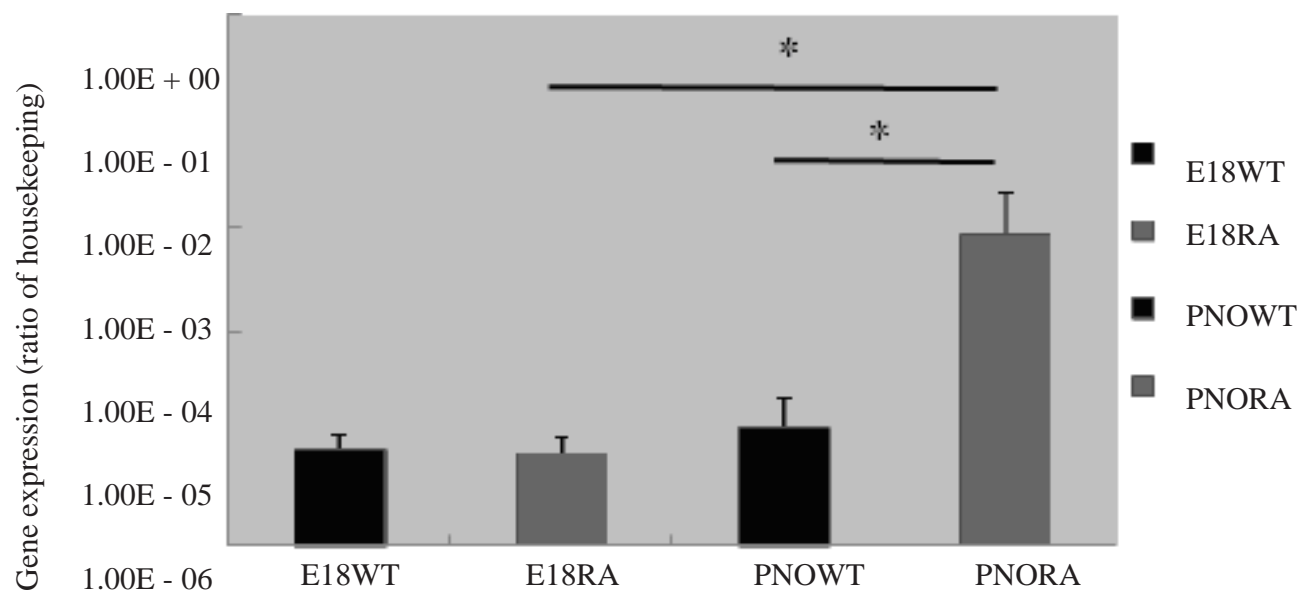

Level of GSK-3 $\beta$ expression

Figure1. Expression of GSK-3 $\beta$ in wild type palatogenesis and RA-induced cleft palate during peinatal stage E18 WT: Wild type palatogenesis at E18, E18 RA: RA-induced palate cleft at E18, PN0 WT: Wild type palatogenesis at PN0 PN0 RA: RA-induced palate cleft at PN0, *: $\mathrm{P}<0.05$ significance by the comparative $\mathrm{C}_{\mathrm{T}}\left(2^{-\triangle \Delta}{ }^{\mathrm{CT}}\right)$ method

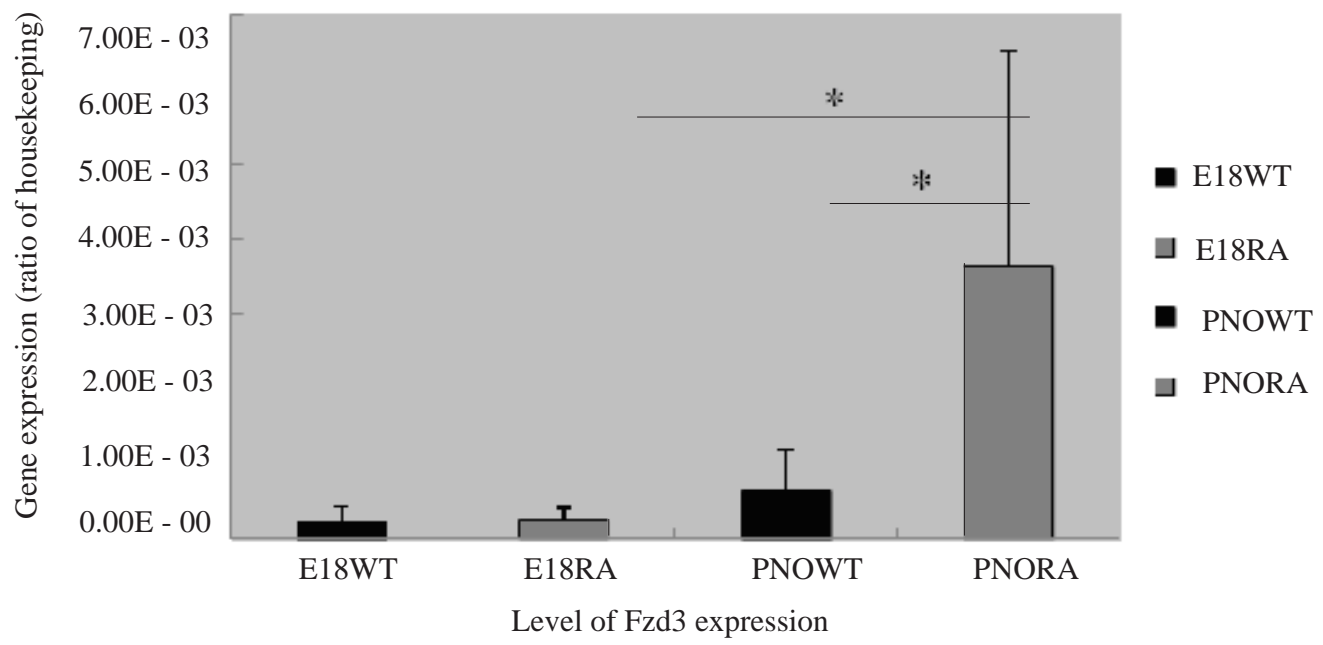

Figure2. Expression of Fzd3 in wild type palatogenesis and RA-induced cleft palate during peinatal stage E18 WT: Wild type palatogenesis at E18, E18 RA: RA-induced palate cleft at E18, PN0 WT: Wild type palatogenesis at PN0 PN0 RA: RA-induced palate cleft at PN0, *: $\mathrm{P}<0.05$ significance by the comparative $\mathrm{C}_{\mathrm{T}}\left(2^{\triangle \triangle \mathrm{CT}}\right)$ method

that are encoded by distinct genes: GSK-3 $\beta$ and GSK-3 $\alpha^{5)}$. In the absence of Wnt, cytoplasm $\beta$-Catenin is phosphorylated by GSK-

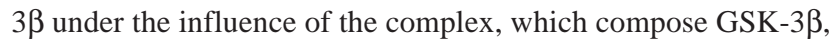
Axin and adenomatous polyposis coli (APC) $)^{4}$. Consequently, GSK-3 $\beta$-mediated phosphorylation $\beta$-Catenin is processed to degradation by ubiquitin-proteasome pathway. Therefore, Gsk3 $\beta$ acts as a negative modulator of $\mathrm{Wnt} / \beta$-catenin signaling. In mice embryonic development, GSK-3 $\beta$ is the essential factor in stem cell survival and palate formation ${ }^{6,7)}$. GSK-3 $\beta$ knockout homozygous mice showed cleft palate, incomplete fusion of the ribs and split sternum ${ }^{8}$.

Frizzled is a family of seven-transmembrane proteins with a characteristic of $\mathrm{G}$ protein-coupled receptor. Frizzled proteins include cysteine-rich domain (CRD) that is conserved in diverse proteins, and need to cooperate LRP to activate the wnt canonical pathways. Frizzled-3 (Fzd3) gene deficiency results in a severe midbrain morphogenesis defect, which consisted of a collapse of brain ventricles, thickening of the neuroepithelium and focal disruption of the ventricular basal lamina ${ }^{9)}$.

As a typical F-box proteins in Eukaryote, Beta-Transducin Repeats-Containing proteins ( $\beta$-TrCP) encoded by the slimb gene, which is a 569 -amino acid (60 $\mathrm{kDa}$ ) regulate protein that belongs to the transducin family. It is noteworthy that Skp1-Cullin-F-box protein (SCF) together with $\beta$-TrCP serves as the substrate recognition subunits for the SCFb-TrCP E3 ubiquitin ligases, which referred the regulation of many important signaling 
Han Liu et al.: Wnt Signals in RA-induced CP at Perinatal Stage



Level of $\beta$-Trcp expression

Figure3. Expression of $\beta$-TrCP in wild type palatogenesis and RA-induced cleft palate during peinatal stage E18 WT: Wild type palatogenesis at E18, E18 RA: RA-induced palate cleft at E18, PN0 WT: Wild type palatogenesis at PN0 PN0 RA: RA-induced palate cleft at PN0, *: $\mathrm{P}<0.05$ significance by the comparative $\mathrm{C}_{\mathrm{T}}\left(2^{-\triangle \Delta c}\right)$ method

molecules. As the F-box protein component of a SCF ubiquitin ligase complex, $\beta$-TrCP is involved in $\beta$-catenin and $\mathrm{I} \kappa \mathrm{B} \alpha$ (inhibitor of NFאB). Recent evidence suggests that $\beta$-TrCP protein is expressed at high levels in brain, lung and liver tissues in mouse embryos $^{10)}$. Moreover, $\beta$-TrCP knockout mice lead to the deficient of the musculoskeletal system in mouse embryos in perinatal mice.

In our current study, the signaling molecules of GSK-3 $\beta$, Fzd3 and $\beta$-TrCP were screened from the palate tissues of the RAinduced CP in mice by the Gene-chip Technology. But, their expression pattern and level in palates during perinatal stages have not been known yet.

\section{Materials and Methods}

\section{Animals}

SPF ICR mice were housed in an animal facility licensed by Dalian Medical University Laboratory Animal Center (Dalian, China, agreement SYXK [Liao] 2008-0002). Animal experiments were supervised by one of the authors who are qualified for experimenting with mice, in compliance with the animal legislation on care and use of laboratory mice. To obtain embryos of a defined gestational age, males and females were housed (males: females=1:2) from $8 \mathrm{Am}$ to $12 \mathrm{Pm}$ and the presence of a vaginal plug was established at embryonic day0 (ED0). All the pregnant mice were randomly divided into two groups: control group (WT group) and RA-treated group (RA group). Pregnant mice in RA group received by gavage a single dose of $100 \mathrm{mg} / \mathrm{kg}$ of RA (Sigma-Aldrich, St. Louis, MO) to establish the mouse model of RA-induced cleft palate on ED10. Pregnant mice in WT group were given corn oil (VO) $(0.2 \mathrm{ml} /$ mouse) at the same time. The palate tissues were harvested from the RA group and WT group at ED18 and postnatal day0 (PN0) respectively.

This study was reviewed and approved by the Ethics Committee of Dalian Medical University.

Table 1. Primers of GSK-3 $\beta$, Fzd3 and $\beta$-TrCP used in quantitative real- time PCR arrays

\begin{tabular}{|c|c|c|c|c|}
\hline Primer & Accession No. & Sequence(5'-3’) & Product(bp) & Tm-cycles \\
\hline \multirow[t]{2}{*}{ GSK-3 $\beta$} & NM_019827 & GGCTGTGTGTTGGCTGAATTGT & & \\
\hline & & GTTGAAGAGTGCAGGTGTGTCT & 354 & $60-35$ \\
\hline \multirow[t]{2}{*}{ Fzd3 } & NM_021458 & TGGGTTGGAAGCAAAAAGAC & & \\
\hline & & CCTGCTTTGCTTCTTTGGTC & 239 & $60-35$ \\
\hline \multirow[t]{2}{*}{$\beta-\operatorname{TrCP}$} & AF_112979 & CCAACTGACATCACССТС & & \\
\hline & & AGCCTGTCTCTGTACTGC & 197 & $60-35$ \\
\hline \multirow[t]{2}{*}{$\beta$-actin } & NM_007393.3 & CATCCGTAAAGACCTCTATGCCA & & \\
\hline & & ATGGAGCCACCGATCCACA & 171 & $60-35$ \\
\hline
\end{tabular}





Figure4. The distribution of GSK-3 $\beta$, Fzd3, $\beta$-TrCP in palate at PN0 after birth.

(A) GSK-3 $\beta$ was detected in the cytoplasm of palatal epithelium in WT group. (B) GSK-3 $\beta$ was detected in RA-induced cleft palatal epithelium, little in the mesenchyme. (C) Fzd3 was detected only in the wild type palatal epithelium. (D) Fzd3 was detected intensely in the RA-induced cleft palatal epithelium. (E) \&(F) $\beta$ TrCP was detected in both of epithelium and mesenchyme of WT and RA groups. (Bar: $10 \mu \mathrm{m}$ )

\section{RNA isolation}

Total RNA was isolated from palate of embryos (ED18) and neonates (PN0) using the Trizol reagent. The quantity and purity of extracted total RNA was assessed by spectrophotometric UV absorbance at 260/280 $\mathrm{nm}$. cDNA were synthesized from total RNA using MyCyclerTM Thermal Cycle system (BIO-RAD, CA, USA). To obtain cDNA, reverse transcription was done in $20 \mu \mathrm{l}$ reaction containing $1.2 \mu$ g total RNA, $5 \times$ PrimeScript $^{\mathrm{TM}}$ Buffer (for Real Time) $4 \mu \mathrm{L}$, PrimeScript ${ }^{\mathrm{TM}} \mathrm{RT}$ Enzyme Mix1 $1 \mu \mathrm{L}$, Oligo dT Primer $1 \mu \mathrm{L}$, Random 6 mers $1 \mu \mathrm{L}$, RNase Free $\mathrm{dH}_{2} \mathrm{O}$ to final to final volume $20 \mu \mathrm{L}$. (TaKaRa, Dalian, China). The reverse transcription reaction consisted of $15 \mathrm{~min}$ at $37^{\circ} \mathrm{C}$ and $5 \mathrm{sec}$ at $85^{\circ} \mathrm{C}$

\section{SYBR Green I-based Real Time PCR}

Complete genome sequences of GSK-3ß (NM_019827), Fzd3 (NM_021458) and $\beta$-TrCP (AF_112979) were retrieved from GenBank (Table 1). $\beta$-actin served as an endogenous control gene. Quantitative real-time PCR was performed by using SYBRPremix Ex Taq ${ }^{\mathrm{TM}}$ II (Perfect Real Time) kit (DRR081A) running on the Thermal Cycler DiceReal Time System (TP800) (TaKaRa, Japan) . The procedure was optimized with regard to concentrations of primers, and denature/extension temperature. The optimized reaction was carried out in a $25 \mu \mathrm{L}$ final reaction volume that 
Han Liu et al.: Wnt Signals in RA-induced CP at Perinatal Stage

contained 2.0 $\mu \mathrm{L}$ cDNA solutions, $12.5 \mu \mathrm{L}$ SYBR Premix Ex Taq ${ }^{\mathrm{TM}} \mathrm{II}(2 \times), 1.0 \mu \mathrm{L}$ PCR Forward Prime, $1.0 \mu \mathrm{L}$ PCR Reverse Primer and $8.5 \mu \mathrm{L} \mathrm{ddH}_{2} \mathrm{O}$ to final volume $25 \mu \mathrm{L}$. The thermal profile for the real-time PCR was $95^{\circ} \mathrm{C}$ for $10 \mathrm{~s}$, followed by 40 cycles of $95^{\circ} \mathrm{C}$ for $5 \mathrm{~s}, 60^{\circ} \mathrm{C}$ for $30 \mathrm{~s}$ with two-step.

\section{Immunohistochemistry}

Primary antibodies that were used are: rabbit anti-human GSK$3 \beta$ antigen polyclonal antibody (Abcam, Cambridge, UK); goat anti-mouse Fzd3 antigen polyclonal antibody (R\&D Systems Inc., Minneapolis, USA) and rabbit anti-human $\beta$-TrCP antigen polyclonal antibody (Santa Cruz Inc., CA, USA).

Paraffin-embedded-4 $\mu$ m-thick tissue sections were prepared. Subsequent to deparaffinization, the sections were hydrated, treated with $3 \% \mathrm{H}_{2} \mathrm{O}_{2}$ for $10 \mathrm{~min}$ and heated in $0.01 \mathrm{M}$ citrate buffer (pH 6.0) and blocked with normal serum. The sections were then incubated with primary antibodies at $4^{\circ} \mathrm{C}$ overnight. The secondary antibody was followed by application of a commercial secondary antibody kit (Kit5010, Maixin Bio, Fujian, China). The immunostaining was performed by immersing the slides in 3, 3'diaminobenzidine under microscopic control. After rinsed with distilled water, the sections were then counterstained with Mayer's hematoxylin and mounted. As negative controls, sections were incubated by PBS instead of the primary antibodies.

\section{Statistical analysis}

Relative quantification analysis was performed using the comparative $\mathrm{C}_{\mathrm{T}}\left(2^{-\Delta \Delta \bullet}\right)$ method ${ }^{11)}$. Validation of the comparative $\mathrm{C}_{\mathrm{T}}\left(2^{-\Delta \Delta \Delta}\right)$ method is used to assess the efficiency of amplification of the target gene and the internal control ( $\beta$-actin). $\Delta \mathrm{CT}=\mathrm{C}_{\mathrm{T}, \mathrm{X}^{-}}$ Ст, $\beta$-actin (X: target gene) was calculated for each cDNA dilution. Association between the RA group and the WT group were assessed by the rank-test. Statistical analysis was analyzed by using SPSS version 17.0 for windows.

\section{Results}

mRNA expression level of GSK-3 $\beta$, Fzd3 and $\beta$-TrCP in WT palatogenesis and $R A$-induced cleft palate during perinatal stage

The mRNA expression level of GSK-3 $\beta$ in wild type palatogenesis (WT group) and RA-induced palate cleft (RA group) were showed in Figure 1. GSK-3 $\beta$ is expressed both at E18 and PN0. The expression level at PN0 is higher than E18 stage both in WT group and RA group, and there is significant difference in RA groups $(\mathrm{P}<0.05)$ at $\mathrm{E} 18$ and $\mathrm{PN} 0$, but not in WT group $(\mathrm{P}>0.05)$. At E18, the expression lever of GSK-3 $\beta$ in the RA group is lower than in the WT group, however, there is no significant difference $(\mathrm{P}>0.05)$; but at $\mathrm{PN} 0$, the expression lever of the RA group is higher than the WT group, showed significantly statistics difference $(\mathrm{P}<0.05)$.
The mRNA expression level of Fzd3 in wild type palatogenesis and RA-induced cleft palate were showed in Figure 2. Fzd3 is also expressed both at E18 and PN0. The expression level at PN0 is higher than E18 stage both in WT group and RA group, and There is significant difference in RA group $(\mathrm{P}<0.05)$ at E18 and PN0, but not in WT group ( $\mathrm{P}>0.05)$. At E18, the expression lever of Fzd3 in the RA group is higher than the WT group, however, there is no significant difference $(\mathrm{P}>0.05)$; but at PN0, the expression lever of the RA group is higher than the WT group, showed significantly statistics difference $(\mathrm{P}<0.05)$.

The mRNA expression level of $\beta$-TrCP in wild type palatogenesis and RA-induced palate cleft were showed in Figure 3. $\beta$-TrCP is expressed both at E18 and PN0 stages. There is no difference between E18 and PN0 in RA group $(\mathrm{P}>0.05)$, but in WT group there is significant difference $(\mathrm{P}<0.05)$. At E18, the expression lever of the RA group is lower than the WT group, showed significantly statistics difference $(\mathrm{P}<0.05)$; At PN0, the expression lever in the RA group is higher than the WT group reversely, showed significantly statistics difference $(\mathrm{P}<0.05)$.

\section{Distribution of GSK-3 $\beta$, Fzd3 and $\beta$-TrCP proteins in palate at PNO after birth}

GSK-3 $\beta$ was briefly expressed in the cytoplasm of palatal epithelium cells of oral side in the WT group(Figure 4A). In RA group, the expression of GSK-3 $\beta$ was observed in the oral side palatal epithelial cells, particularly in medial edge epithelium (MEE), and little in the mesenchymal cells(Figure 4B). Fzd3 protein was located only in the palatal epithelium, but not in mesenchyme of WT group (Figure 4C). In the RA group, Fzd3 showed more intense expression in palatal epithelium (Figure 4D). $\beta$-TrCP was expressed in both in the palatal epithelial cells of oral side and mesenchymal cells. In mesenchyme of WT group, intense staining can be observed in osteoblasts (Figure 4E). Whereas, in the RA group, positive staining of $\beta$-TrCP located in both of the oral and nasal side palatal epithelial cells and underlying mesenchymal cells, especial focus on the MEE(Figure $4 \mathrm{~F})$.

\section{Discussion}

Palate development depends on a complex process regulated by the networks of growth factors, extracellular matrix (ECM) and cellular surface receptor. The loss of regulation may bring to the abnormalities of palatal fusion, and ultimately causes a cleft palate formation. Recently, normous progress of research have been done in the palatal formation and development regulated by these genes during early embryonic stage, however, the spatiotemporal expression pattern and interaction of these genes are still unclear during later embryonic day and postnatal day of palate development.

The Wnt family-mediated signaling transduction pathways 
J.Hard Tissue Biology Vol. 21(2):173-180, 2012

plays pivotal role in regulating multiple events of embryonic development. These linked to the embryonic axial development, cell polarity and cell fate. GSK-3 $\beta$ is the negative regulatory modulator of the Wnt/ $\beta$-catenin signing pathway ${ }^{12)}$. Liu et al demonstrated that GSK-3 $\beta$ homozygous null mice display cleft palate, incomplete fusion of the ribs and bifid sternum ${ }^{7)}$ in 2007. He et al. demonstrated determine that GSK-3 $\beta$ and is essential in palatogenesis by setting up a GSK-3 $\beta$ conditional knockout mice model, and it is particularly located in the medial edge epithelium (MEE) of palate and plays a role in the elevation palatal shelves. Their data also showed significantly increased apoptotic cells in the palatal epithelium of the GSK3ß-/- palate. All these results indicate that GSK-3 $\beta$ is a key regulator of cell proliferation and survival during palatogenesis ${ }^{13)}$. In our experiment, although GSK$3 \beta$ is expressed at high levels in both the RA group and the WT group during PN0 stage, but the RA group showed significantly higher than the WT group ( $\mathrm{P}<0.05)$, this result reveals that as a inhibitor of the Wnt pathway, GSK-3 $\beta$ up-regulation at the mRNA levels in the palatal epithelium of RA-induced cleft palate after birth, it implies that a tendency to suppressive effect of retinoic acid on wnt signaling pathways via modulating GSK-3 $\beta$.

Slimb (the homologous F-box proteins of $\beta$-TrCP in Drosophila) can inhibit the Wnt signaling pathway, and Slimb-knockout leads to Wnt gene over-expression in Drosophila. This inhibiting effect of $\beta$-TrCP on Wnt signaling pathway have the characteristics of highly conserved, meanwhile, there was no difference in quantitative species. The factors downstream of Wnt signaling pathways can be hydrolyzed by Slimb gene in Drosophila and showed distinctively expression in the different tissues. In the mammals, studies have been shown that the overexpression of Slimb/ $\beta$-TrCP can cause the decrease expression of wnt, ptch1and ptch2 in tooth germ cells and meanwhile diminish proliferative capacity of the epithelium of the enamel and the dental mesenchyme cells ${ }^{14,15)}$. With the assistance of Slimb/ $\beta$-TrCP, phosphorylated cytoskeletal protein- $\beta$-catenin has a tendency to undergo proteolysis and subsequent degraded by the $26 \mathrm{~S}$ proteasome. Therefore, $\beta$-catenin which cannot binds the TCF transcription factor ultimately inhibiting target genes transcription. To our knowledge, role of $\beta$-TrCP in palatogenesis and cleft palate has never been reported in literature. Thus, our data in conjunction with other findings may help to explain that the expression of $\beta$ TrCP confine the extent of Wnt signaling pathway in palatogenesis during perinatal stage. In our result, $\beta$-TrCP in the RA group is higher than the WT group at the PN0 stage, with a significant difference $(\mathrm{P}<0.05)$, it implies that retinoic acid up-regulated $\beta$ TrCP expression after brith, then suppress Wnt signaling pathway. Deardorff et al. demonstrated that Fzd3 specifically induces the neural crest migration. Fzd3 overexpression can disrupt the convergent extension in xenopus gastrula/neurula ${ }^{16)}$. Some researchers proposed that Fzd3 protein has been limited in anterior neuroectodermal endoplasmic reticulum (ER) by Shisa ${ }^{17)}$, meanwhile, activity against Wnt/Fz signalling. In the research of Wnt/Frizzled family members mediate olfactory sensory neuron (OSN) axon extension, the researcher found that Wnt5a, Wnt4, Wnt11 and Fz3 showed highly expressed on both the olfactory epithelium (OE) and olfactory bulb (OB) ${ }^{18)}$. In the present study direct evidence for Fzd3 in cleft palate is still lacking. Therefore, in this paper, we reported that Fzd3 protein is expressed in the palatal epithelium in WT group and RA group. Based on these findings, we postulate that Fzd3 may play proval role in the palatal epithelium, that is to say, Fzd3 mediates the PCP pathway. Fzd3 is Wnt5a receptor; Wnt5a can iactivate canonical Wnt/ $\beta$-catenin signaling pathways through the interaction of Wnt/ $\beta$-catenin canonical and Wnt/Ca ${ }^{2+}$ non-canonical signaling pathways. Our data showed the mRNA expression level of Fzd3 at pN0 stage is higher than E18 stage ( $\mathrm{P}>0.05)$ and suggest Fzd3 may act as the receptor of Wnt5a, indirectly inhibit the activity of Wnt canonical signaling pathway in palatogenesis during postnatal stage.

In this study, we explored the mRNA expression of signaling molecules related Wnt signaling pathway in palatogenesis and RA-induced cleft palate during perinatal stage and the location after brith. However, in the cleft palate induced by retinoic acid during perinatal stage, it still keeps unknown that how retinoic acid up-regulates the mRNA expression of GSK-3 $\beta$, $\beta$ TrCP and Fzd3; The pathways by which Fzd3 involve in which Wnt signalling pathways in the spatiotemporal changes of palate; whether or not specific acts on some of wnt proteins, and so no. If the mechanisms of signaling molecule related Wnt signaling pathway in the organ development during both of the embryonic and adult stages are clear, it will be useful for repairing the tissue defects caused by congenital disease by effective stem cell therapy.

\section{Acknowledgements}

This study was supported by National Natural Science Foundation of China (No. 30973349); The Ph.D. Programs Foundation of Ministry of Education of China (No.20092105110006); The Project supported by the Higher Education Institutions of Liaoning Educational Committee (No.2008166). Han Liu and Hui Wu gives equal distribution on this article and Grant for Supporting Project for Strategic Research from MEXT (2008-2012), S0801032.

\section{References}

1. Wang R, Cong W, Huang H-T and Xiao J. Expression of wnt signaling pathway components in RA-induced cleft palate mouse. J Hard Tissue Biol 14: 241-242, 2005

2. Grigoryan T, Wend P, Klaus A and Birchmeier W. Deciphering the function of canonical Wnt signals in development and disease: conditional loss- and gain-offunction mutations of beta-catenin in mice. Genes Dev 22: 
Han Liu et al.: Wnt Signals in RA-induced CP at Perinatal Stage

2308-2341, 2008

3. Wu D and Pan W. GSK3: A multifaceted kinase in Wnt signaling. Trends Biochem Sci 35: 161-168, 2010

4. Cadiqan KM and Liu YI. Wnt signaling: Complexity at the surface. J Cell Sci 119: 395-402, 2006

5. Doble BW and Woodgett JR. GSK-3: tricks of the trade for a multi-tasking kinase. J Cell Sci 116: 1175-1186, 2003

6. Hoflich KP, Luo J, Rubie EA, Tsao MS, Jin O and Woodgett JR. Requirement for glycogen synthase kinase-3beta in cell survival and NF-kappa B activation. Nature 406: 86-90, 2000

7. Liu KJ, Arron JR, Stankunas K, Crabtree GR and Longaker MT. Chemical rescue of cleft palate and midline defects in conditional GSK-3beta mice. Nature 446: 79-82, 2007

8. Stuebner S, Faus-Kessler T, Fischer T, Wurst W and Prakash N. Fzd3 and Fzd6 deficiency results in a severe midbrain morphogenesis defect. Dev Dyn 239: 246-260, 2010

9. Kudo Y, Guardavaccaro D, Santamaria PG, Koyama-Nasu R, Latres E, Bronson R, Yamasaki L and Pagano M. Role of F-box protein betaTrcp1 in mammary gland development and tumorigenesis. Mol Cell Biol 24: 8184-8194, 2004

10. Spiegelman VS, Tang W, Chan AM, Igarashi M, Aaronson SA, Sassoon DA, Katoh M, Slaga TJ and Fuchs SY. Induction of homologue of Slimb ubiquitin ligase receptor by mitogen signaling. J Biol Chem 277: 36624-36630, 2002

11. Livak KJ and Schmittgen TD. Analysis of relative gene expression data using real-time quantitative PCR and the 2(-Delta Delta C (T)) Method. Methods 25: 402-408, 2001

12. Ciaraldi TP, Oh DK, Christiansen L, Nikoulina SE, Kong AP, Baxi S, Mudaliar S and Henry RR. Tissue-specific expression and regulation of GSK-3 in human skeletal muscle and adipose tissue. Am J Physiol Endocrinol Metab 291: E891-E898, 2006

13. He F, Popkie AP, Xiong W, Li L, Wang Y, Phiel CJ and Chen Y. Gsk3 beta is required in the epithelium for palatal elevation in mice. Dev Dyn 239: 3235-3246, 2010

14. Smith AJ. Tooth tissue engineering and regeneration-a translational vision. J Dent Res 83: 517, 2004

15. Yen AH and Sharpe PT. Regeneration of teeth using stem cell-based tissue engineering. Expert Opin Biol Ther 6: 916, 2006

16. Deardorff MA, Tan C, Saint-Jeannet JP and Klein PS. A role for frizzled3 in neural crest development. Development 128: 3655-3663, 2001

17. Yamamoto A, Nagano T, Takehara S, Hibi M and Aizawa S. Shisa promotes head formation through the inhibition of receptor protein maturation for the caudalizing factors, Wnt and FGF. Cell 120: 223-35, 2005

18. Rodriguez-Gil DJ and Greer CA. Wnt/Frizzled family members mediate olfactory sensory neuron axon extension. J Comp Neurol 511: 301-317, 2008 
J.Hard Tissue Biology Vol. 21(2):173-180, 2012 